\title{
Experimental Setup to Measure Dispersion in Optical Fiber Devices
}

\author{
Luis Alberto Rodríguez Morales, Iván Armas Rivera, Georgina Beltrán Pérez \\ Benemérita Universidad Autónoma de Puebla, Puebla, \\ Mexico
}

\begin{abstract}
Some of the different factors that lower performance of the optical fiber and experimental components are attenuation and dispersion. Some are intrinsic to the material and cannot be deleted, but can be compensated, so it is therefore necessary to quantify the chromatic dispersion. This paper presents a method for measuring the coefficient of dispersion in a WDM and an optical isolator, caused by the temporal broadening of a pulse due to the different speeds that are in the optical frequencies of said pulse. The Mach-Zehnder interferometer has many applications, in this paper it's one, in which a WDM and Optical insolator are analyzed, the data obtained by the method were channeled by a measured optical spectrum (OSA for its acronym) and then analyzed on the computer with a program previously done in Matlab. The results clearly show that the dispersion can be measured with high accuracy not only optical fibers but also in other optical devices.
\end{abstract}

Keywords: optics fiber, dispersion, WDM, optical isolator.

\section{Introduction}

Dispersion is a quite important parameter in systems based on optical fiber since it affects the original temporal profile with which the data have been sent. It also reduces the number of channels that can be transmitted simultaneously. Therefore, it is necessary to consider the dispersion provoked by each optical component of the system.

The chromatic dispersion in an optical fiber is originated by the effective refractive index variation as a wavelength function. This parameter can affect the bandwidth of a high speed optical transmission system, broadening the pulse and causing errors in the received information. Among the most important methods used to measure chromatic dispersion in long length optical fibers there can be mentioned the following: the pulse-delay method which measures the difference between modes in optical pulses with various wavelengths. Even though this is a very simple and inexpensive technique, pulse broadening due to chromatic dispersion degrades its accuracy, since this makes difficult to accurately determine the arrival time of a pulse [L. G. Cohen, et al.]. Another technique is the modulation phase-shift techniques that use the time-of-flight method to measure chromatic dispersion. This method is accurate and repeatable. Phase-modulated optical signals are transmitted through a 
long sample of an optical fiber, and their phase delays are measured as a function of the wavelength.

However, this technique has several drawbacks. First, the accuracy of this method is restricted when the selected wavelength is far from the reference wavelength, with large phase differences between the reference and test-sample arms generating ambiguities in phase-shift measurement. Second, it requires a complicated experimental setup and expensive equipment such as a high-speed optical modulator and an optical tunable filter. Finally, it cannot measure the chromatic dispersion of a short fiber sample [L. G. Cohen, et al], [J. Brendel, et al]. In the present work an experimental arrangement to measure chromatic dispersion in optical fibers of short length using a configuration based on a Mach-Zehnder interferometer is proposed. The device under test (DUT) is located in one arm while an air arm reference is used to obtain the spectral interferogram. This technique is capable of performing a sweep of several wavelengths in milliseconds, besides it is quite simple to implement. The versatility of this experimental setup allows the easy replacement of the fiber under test for its characterization. With this, we might measure the chromatic dispersion in nonconventional fibers which do not have datasheet available about their dispersion coefficient.

The obtained results with the present technique can be used to optimize communication systems based in optical elements composed by fiber, increasing the transmission data capacity and decreasing the error rate in the reception.

\section{Experimental Setup}

Our experimental setup for the measurement of material dispersion is a MachZehnder interferometer, this arrangement consists in two arms, where one arm is formed by the DUT, the other one is made up of air. This interferometer is powered by a broadband source ( $1550 \mathrm{~nm}$ laser-diode, Polarizers plates, two 50/50 couplers, and two lenses to collimate the beam arm through which the beam travels in air. The Fig 1 shows the experimental setup.

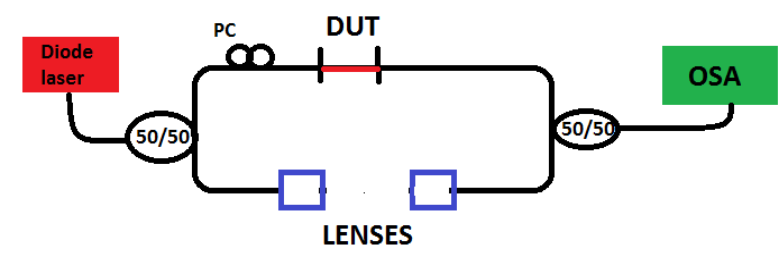

Fig. 1. Experimental arrangement to measure chromatic dispersion in single mode optical fiber based on a Mach-Zehder interferometer.

\section{Theoretical Considerations}

The intensity is related to the field as follows: " $\mathrm{I}=|E|^{2}$ ", if the explicit dependencies are omitted to simplify the expression intensity as interferometer this is expressed like: 


$$
I=I_{0}\left\{1+2 \alpha(\alpha-1)\left[1+\cos \left(\beta(\lambda) L-\beta_{0} L_{0}\right) .\right.\right.
$$

Then only remains us to obtain the phase of the cosine term to obtain an estimate of " $\beta(\lambda)$ ", if we assume that this process of phase extraction has already been done, we can do a change of variable " $\lambda$ " by " $f$ " expressing the relative phase as:

$$
\phi(f)=\beta(f) L-\beta_{0} L_{0},
$$

where $\beta(f)$ is the propagation constant of the transmitted light on the test device. The delay time associated with the reference arm in the interferometer is defined as $\tau_{0=\frac{L_{0}}{C_{0}}}$ ,so the relative phase is expressed as:

$$
\phi(f)=\beta(f) L-2 \pi \tau_{0} f .
$$

Differentiating the phase with respect to the optical frequency are:

$$
\frac{1}{2 \pi} \frac{d \phi(f)}{d f}=\tau_{g}(f)-\tau_{0}
$$

where

$$
\tau_{g}(f)=\frac{L}{v_{g}(f)}=\frac{L}{2 \pi} \frac{d \beta(f)}{d f} .
$$

Then the derivative of delay group respect to the wavelength provides the coefficient of dispersion value. So we made a variable change from " $\mathrm{f}$ " to " $\lambda$ ", then calculate the phase's second derivate to have it.

$$
D=\frac{1}{L} \frac{d \tau_{g}}{d \lambda_{0}}
$$

\section{Results}

To obtain the dispersion value, various interferograms were measured changing the optical path difference in order to determine the optimum optical path. The dispersion coefficient is calculated using just the interferogram obtained with such optical path. To obtain more precise and reliable values is necessary to take various measurements with the same parameters and to calculate an average.

The cross correlation interferogram obtained directly from the OSA within a range of $200 \mathrm{~nm}$ is shown in Figure 2 and Figure 3. It can be noted that the plot shape resembles that for the diode laser, however it is modulated depending on the optical path, which can be changed with the translation stages. 


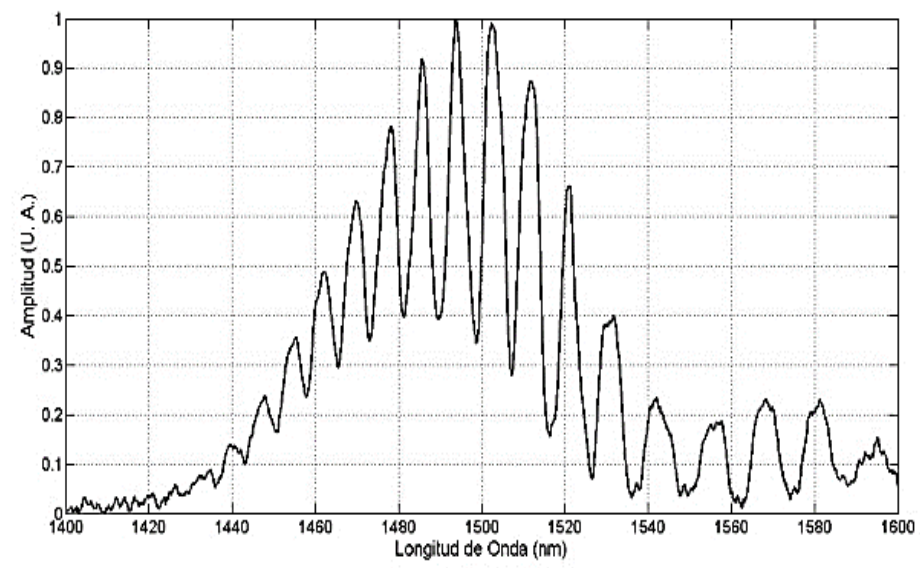

Fig. 2. WDM interferogram.

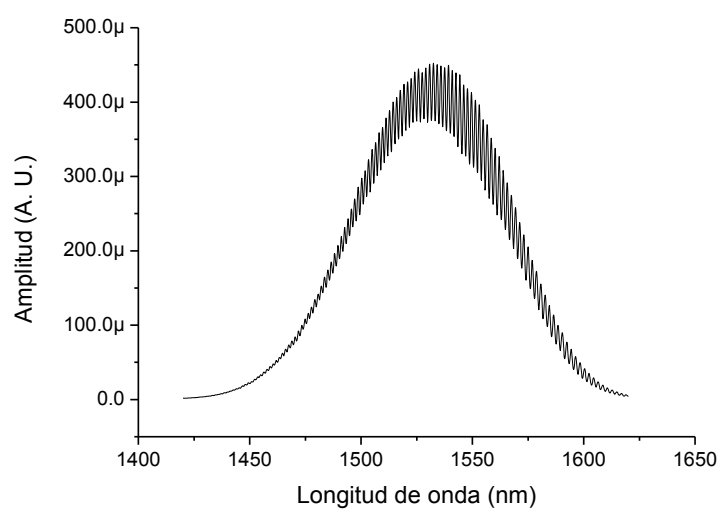

Fig. 3. Optical isolator interferogram.

Unlike the results obtained in previously reported works [J. Y. Lee, et al.], where the phase is calculated by measuring the separation between positive fringe peaks from the spectral interferogram, in this work we measure the phase behavior in function of wavelength using Fourier transformation [Mitsuo Takeda, et al.]. We use a Fast-FourierTransformed algorithm (FFT) to filter unwanted information such as slow spatial variation, compared with the carrier frequency, and amplitude variations. Again, using the FFT algorithm, we apply the inverse Fourier transform to the filtered signal to obtain the phase simply dividing the imaginary part by the real part. This phase is wrapped, therefore is necessary to apply an algorithm to unwrap it. This dependence is in terms of the wavelength, however it is necessary to perform a variable change since equation 2 is in terms of frequency. 


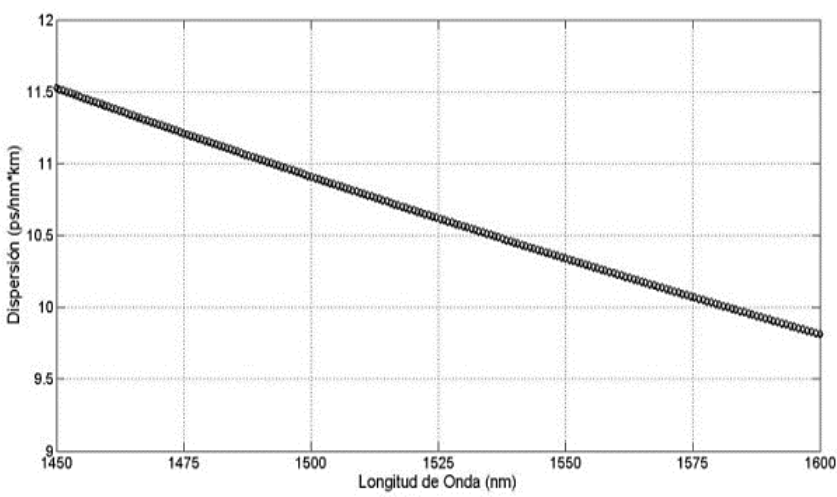

Fig. 4. WDM dispersion curve.

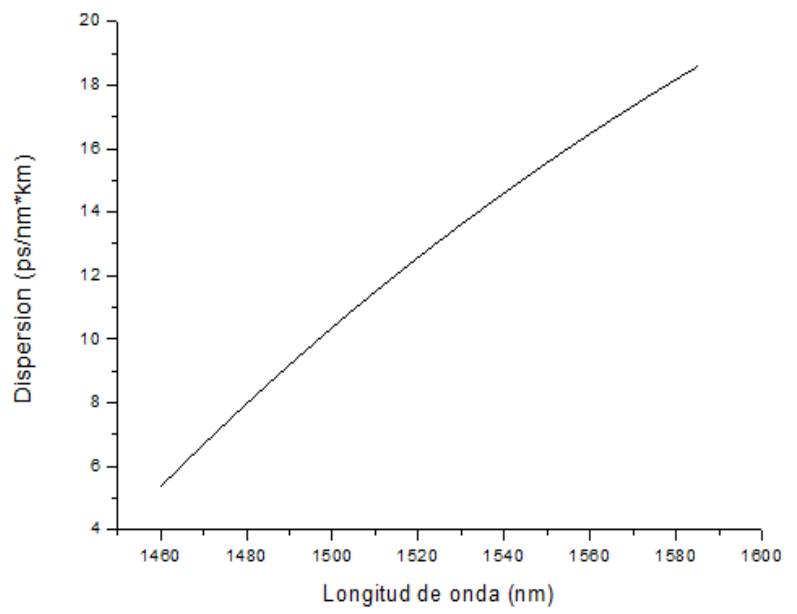

Fig. 5. Optical isolator dispersion curve.

Once the phase behavior was obtained it was performed a fitting using a third order polynomial.

Based on equations 3 and 5, the first derivative respect to the frequency of this expression was calculated to obtain the group delay, and the second derivative respect to the wavelength was calculated to obtain the dispersion coefficient. With this procedure the results shown in Fig. 4 and Fig. 5 were obtained. The shown dispersion values were experimentally obtained using two different wavelength resolutions $(0.5$ and $1 \mathrm{~nm}$, dotted line and dashed line, respectively).

\section{$5 \quad$ Discussion and Conclusions}

Nowadays in the Laboratory of Electronics and Optoelectronics, LEyO, we have an interferometric system to measure of the coefficient of dispersion in optical fibers in 
a wavelength range of 1520-1570 nm. This system is based on a Mach-Zehnder interferometer, where the reference arm light travels through the air.

The biggest advantage of this system is that it only measures for a fiber length of several tens of centimeters is required in addition to the great simplicity to change the fiber under test as temporary connectors are used for connection to the system.

In this work an experimental arrangement to evaluate dispersion in WDM and optical isolator were developed. At the umbral of $1550 \mathrm{~nm}$, which is the umbral of the communication, the WDM has a dispersion about $10 \mathrm{ps} / \mathrm{nm} * \mathrm{Km}$ and the optical isolator about $14 \mathrm{ps} / \mathrm{nm}^{*} \mathrm{Km}$.

The contribution to the dispersion value due to the optical elements that compose the experimental arrangement has been neglected owing to the fact that their physical dimensions are much shorter that the DUT. On the other hand it is thought that the same effects of each element are present in both arms of the interferometer and that their contribution is quite small.

Acknowledgments. This word was supported by project VIEP-BUAP BEPGEXC14-I and PRODEP 2014.

\section{References}

1. Armas-Rivera, I.: Método para la medición de la dispersión en fibras ópticas por medio de un interferómetro de Mach-Zehnder. Tesis de licenciatura, México, BUAP (2011)

2. Saleh, B. E. A., Teich, M. C.: Fundamentals of Photonics. Wiley, Second Edition, (2007)

3. Brendel, J., Zbinden, H., Gision, N.: Measurement of chromatic dispersion in optical fibers using pairs of correlated photons. Opt. Commun., 151, pp. 35-39 (1998)

4. Lee, J. Y., Kim, D. Y.: Versatile chromatic dispersion measurement of a single mode fiber using spectral white light interferometry. Republic of Korea (2006)

5. Cohen, L. G.: Comparison of single-mode fiber dispersion measurement techniques. J. Lightwave Technol., 3, pp. 958-966 (1985)

6. Cohen, L. G., Chinlon, L.: Pulse delay measurements in the zero material dispersion wavelength region for optical fibers. Appl. Opt., 16, pp. 3136-3139 (1977)

7. Takeda, M., Ina, H., Kobayashi, S.: Fourier-transform method of fringe-pattern analysis for computer-based topography and interferometry. Tokyo, Japan (1981)

8. Hui, R., O’Sullivan, M.: Fiber Optic Measurement Techniques. Elsevier Academic Press (2009) 